\title{
Electrochemical Preparation of Cobalt Nano-particles in an Ionic Liquid ${ }^{\dagger}$ Yasushi KATAYAMA,* Ryuta FUKUI, and Takashi MIURA
}

\author{
Department of Applied Chemistry, Faculty of Science and Technology, Keio University, \\ 3-14-1 Hiyoshi, Kohoku-ku, Yokohama, Kanagawa 223-8522, Japan \\ * Corresponding author: katayama@applc.keio.ac.jp
}

\begin{abstract}
Cobalt nano-particles have been prepared by reducing divalent cobalt species in an ionic liquid, 1-butyl-1methylpyrrolidinium bis(trifluoromethylsulfonyl)amide at room-temperature. Potentiostatic cathodic reduction of divalent cobalt species was conducted on a platinum mesh electrode at $-2.0 \mathrm{~V} \mathrm{vs}$. $\mathrm{Ag} / \mathrm{Ag}(\mathrm{I})$ in the ionic liquid, resulting in the change of color of the ionic liquid from purple to black. The transmission electron microscope observation of the ionic liquid after the potentiostatic cathodic reduction showed formation of cobalt nano-particles with the diameters from 2 to $10 \mathrm{~nm}$.
\end{abstract}

(c) The Electrochemical Society of Japan, All rights reserved.

Keywords : Ionic Liquid, Nano-particle, Cobalt

\section{Introduction}

Ionic liquids have been expected as the promising electrolytes for electrodeposition of various metals since some of aprotic ionic liquids have such favorable properties as wide usable temperature range, wide electrochemical potential window, negligible vapor pressure, and acceptable intrinsic ionic conductivity. ${ }^{1}$ We have already reported the electrodeposition of iron, ${ }^{2}$ cobalt, ${ }^{3-5}$ nickel, ${ }^{6-8}$ tin, ${ }^{,, 10}$ and silver ${ }^{11-13}$ from an amide-type ionic liquid, 1-butyl-1-methylpyrrolidinium bis(trifluoromethylsulfonyl)amide (BMPTFSA). In the cases of tin and silver, the crystalline metals could be obtained by cathodic reduction of their ions dissolved in the ionic liquid at room-temperature. On the other hand, it was difficult to obtain the crystalline phases of iron, cobalt and nickel by electrodeposition in the ionic liquid at room-temperature. In these cases, the obtained electrodeposits gave no X-ray diffraction (XRD) peaks, indicating the grain sizes of the electrodeposits were too small. However, when the temperature was elevated more than $100^{\circ} \mathrm{C}$, the crystalline metals could be deposited electrochemically. Thus, formation of amorphous-like deposits could be explained by slow crystal growth of these metals in the ionic liquid at roomtemperature.

The reduction of metal species in the ionic liquid sometimes results in the change of appearance of the ionic liquids. In case of nickel, ${ }^{8}$ for instance, the yellow transparent ionic liquid changed into the black opaque one after cathodic reduction of nickel species in the ionic liquid. Formation of nickel nano-particles was confirmed by the observation of the ionic liquid by transmission electron microscope (TEM). Formation of silver nano-particles has been also confirmed in the same ionic liquid. ${ }^{13}$ These results indicate the slow crystal growth in the ionic liquid may cause the formation of the metal nano-particles, which are not adhered on electrodes but dispersed in the ionic liquid. Therefore, ionic liquids are expected to be utilized for production of metal nano-particles by a simple electrochemical process. In the present study, electrochemical formation of cobalt nano-particles has been investigated in BMPTFSA.

Presented at the 4th Asian Conference on Molten Salt Chemistry and Technology

\section{Experimental}

BMPTFSA was prepared by the metathesis reaction of LiTFSA (Kanto Chemical) and $\mathrm{BMPBr}$, which was prepared by the reaction of butyl bromide (Tokyo Chemical Industry) and 1-methylpyrrolidine (Tokyo Chemical Industry). BMPTFSA was purified by extraction into dichlorometane in the presence of water, separated by evaporation, and finally dried under reduced pressure at $120^{\circ} \mathrm{C}$. The water content in purified BMPTFSA was confirmed to be below 10 ppm by Karl Fischer titration (Metrohm, $831 \mathrm{KF}$ Coulometer). $\mathrm{Co}$ (TFSA) $)_{2}$ was prepared by the reaction of $\mathrm{CoCO}_{3}$ (Kanto Chemical) and HTFSA (Morita Chemical Industry) aqueous solution. Water in as-prepared $\operatorname{Co}(\text { TFSA })_{2}$ was eliminated by drying under reduced pressure at $120^{\circ} \mathrm{C}$. Glassy carbon (GC-20S, Tokai Carbon) or a Pt mesh (Sanwa Kinzoku) was used as a working electrode. Co (Nillaco) was used as a counter electrode. A silver wire (Sanwa Kinzoku) immersed in $0.1 \mathrm{M} \mathrm{AgCF}_{3} \mathrm{SO}_{3} / \mathrm{BMPTFSA}$ was used as a reference electrode. The inner electrolyte of the reference electrode was separated from the main electrolyte by a porous glass (Vycor). All the electrochemical measurements were conducted in a argon-filled glove box with a continuous gas purification apparatus (Miwa Seisakujyo Co. Ltd., DB0-1K-SH) with the aid of a potentiostat/galvanostat (Hokuto Denko, HABF-501). The TEM samples were prepared by dropping the ionic liquid sample on TEM grids (Okenshoji). The grids were washed with acetonitrile and dried. TEM images were taken by Tecnai Spirit (FEI).

\section{Results and Discussion}

Figure 1 shows the cyclic voltammogram of a glassy carbon electrode in BMPTFSA containing $100 \mathrm{mM} \mathrm{Co(TFSA})_{2}$ at roomtemperature. A cathodic peak around $-2.0 \mathrm{~V}$ and an anodic peak around $0.0 \mathrm{~V}$ can be assigned to the cathodic deposition and the anodic dissolution of cobalt, respectively. ${ }^{3,4}$ The cathodic peak potential was much more negative than the anodic peak potential, indicating the overpotential for deposition of cobalt is fairly large. The large overpotential is caused by the inhibition of surface processes on the negatively polarized electrode, on which bulky $\mathrm{BMP}^{+}$are accumulated in order to compensate the negative charge on the electrode. ${ }^{13}$

Figure 2 shows the current transient during the potentiostatic cathodic reduction at $-2.0 \mathrm{~V}$ on a Pt mesh electrode in BMPTFSA 


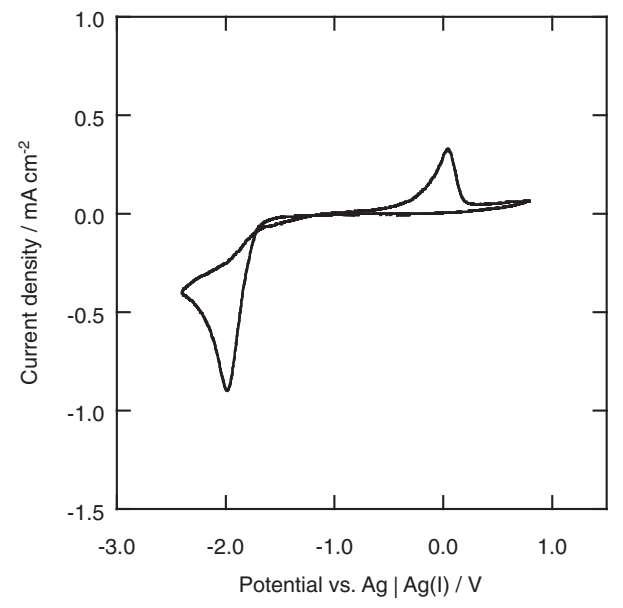

Figure 1. Cyclic voltammogram of a glassy carbon electrode in BMPTFSA containing $100 \mathrm{mM} \mathrm{Co}(\mathrm{TFSA})_{2}$ at $25^{\circ} \mathrm{C}$. Scan rate: $10 \mathrm{mV} \mathrm{s}^{-1}$.

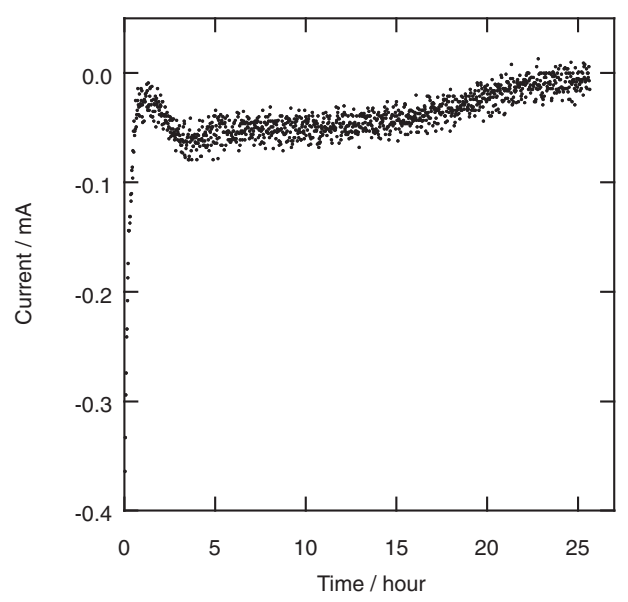

Figure 2. Current during the potentiostatic cathodic reduction at a Pt mesh electrode in BMPTFSA containing $100 \mathrm{mM} \mathrm{Co(TFSA)})_{2}$ at $25^{\circ} \mathrm{C}$. Applied potential: $-2.0 \mathrm{~V}$.

containing $100 \mathrm{mM} \mathrm{Co(TFSA})_{2}$ at room-temperature. The counter electrode was separated from the working electrode compartment with a glass filter using a two-compartment cell. The steady-state current of about $-0.05 \mathrm{~mA}$ was attained after applying the constant potential. The current diminished gradually with the elapse of time, reflecting a decrease in the bulk concentration of $\mathrm{Co}(\mathrm{II})$ in BMPTFSA. The purple transparent ionic liquid was turned to the black opaque one after the potentiostatic cathodic reduction for $25 \mathrm{~h}$, while the black and less adhesive Co was also deposited on the platinum mesh electrode. The TEM image of the ionic liquid clearly showed the formation of nano-particles dispersed in the ionic liquid, as shown in Fig. 3. The sizes of nano-particles were in the range from 2 to $10 \mathrm{~nm}$. Figure 4 shows the EDX spectra of the regions including and excluding the nano-particles in the TEM image. Cobalt was found only in the region including the nano-particles. Since it has been known that Co(II) is reduced to Co at the applied potential, the nano-particles can be identified as metallic cobalt.

The formation of cobalt nano-particles may be explained by the hindrance of crystal growth as a result of the accumulation of $\mathrm{BMP}^{+}$ on the negatively polarized electrode. The electrodeposition of cobalt occurs around $-2.0 \mathrm{~V}$, which is close to the cathodic potential limit of the ionic liquid. Since the electrode is charged negatively at such negative potentials, the cationic species are accumulated at the interface between the electrode and the electrolyte in order

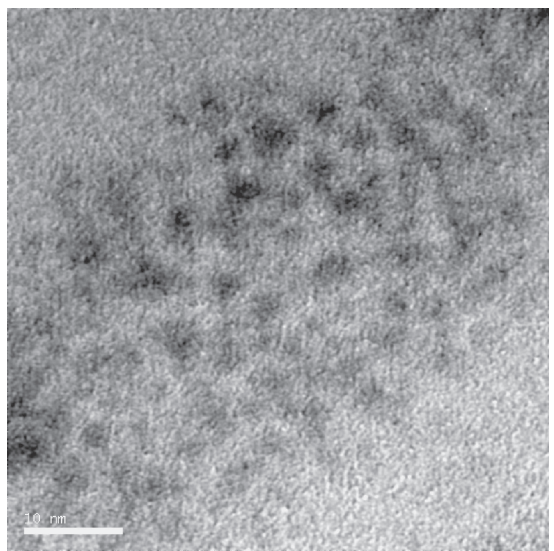

Figure 3. TEM image of the electrolyte after potentiostatic cathodic reduction with a $\mathrm{Pt}$ mesh electrode in BMPTFSA containing $100 \mathrm{mM} \mathrm{Co}(\mathrm{TFSA})_{2}$ at $25^{\circ} \mathrm{C}$. Applied potential: $-2.0 \mathrm{~V}$.
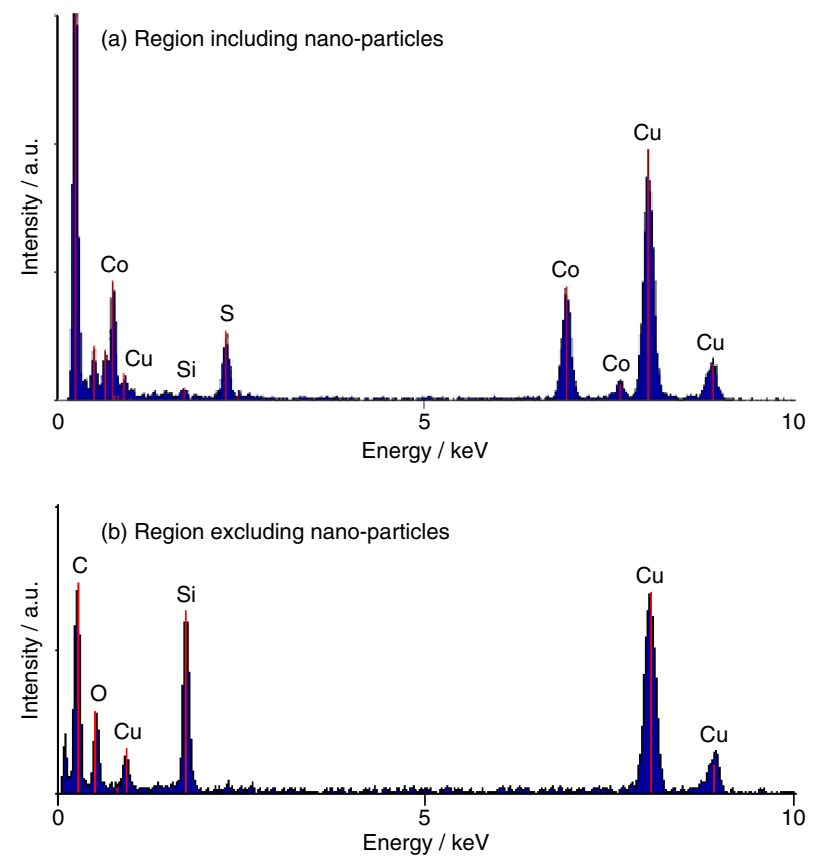

Figure 4. (Color online) EDX spectra obtained in the regions (a) including and (b) excluding nano-particles for the TEM image of the electrolyte after potentiostatic cathodic reduction with a $\mathrm{Pt}$ mesh electrode in BMPTFSA containing $100 \mathrm{mM} \mathrm{Co(TFSA)})_{2}$ at $25^{\circ} \mathrm{C}$. Applied potential: $-2.0 \mathrm{~V}$.

to compensate the excess negative electric charge. The divalent cobalt species in BMPTFSA is considered as an anionic species, $\left[\mathrm{Co}(\mathrm{TFSA})_{3}\right]^{-, 14}$ which can not participate in the charge compensation at the negatively polarized electrode surface. Since $\mathrm{BMP}^{+}$is the only species with a positive charge in the ionic liquid, the electrode surface is expected to be covered mainly by the $\mathrm{BMP}^{+}$layer, which may hinder such surface processes as the crystal growth and the adhesion of deposits. Consequently, it is expected that part of cobalt metal does not adhere to the surface of the electrode or deposit and disperses as nano-particles in the ionic liquid, which is known to have an ability to disperse nano-particles. ${ }^{15}$

\section{Conclusions}

Cobalt nano-particles have been successfully prepared by reducing divalent cobalt species in BMPTFSA at room-temperature. 
The formation of cobalt nano-particles may be explained by the slow crystal growth of cobalt metal due to the accumulation of $\mathrm{BMP}^{+}$on the negatively charged electrode surface and the stabilization of the nano-particles by the ionic liquid. Preparation of the nano-particles of various metals is expected to be possible using this simple electrochemical method. Furthermore, the dispersed metal nanoparticles may be utilized as catalysts for various chemical processes.

\section{Acknowledgments}

Part of this study was financially supported by MEXT-Supported Program for the Strategic Research Foundation at Private Universities, 2012-2016.

\section{References}

1. H. Ohno, Electrochemical Aspects of Ionic Liquids, 2nd Edition, Wiley, Inc., Hoboken, NJ (2011)
2. M. Yamagata, N. Tachikawa, Y. Katayama, and T. Miura, Electrochemistry, 73, 564 (2005).

3. R. Fukui, Y. Katayama, and T. Miura, Electrochemistry, 73, 567 (2005).

4. Y. Katayama, R. Fukui, and T. Miura, J. Electrochem. Soc., 154, D534 (2007).

5. R. Fukui, Y. Katayama, and T. Miura, Electrochim. Acta, 56, 1190 (2011).

6. Y.-L. Zhu, Y. Kozuma, Y. Katayama, and T. Miura, Electrochim. Acta, 54, 7502 (2009).

7. Y.-L. Zhu, Y. Katayama, and T. Miura, Electrochim. Acta, 55, 9019 (2010).

8. Y.-L. Zhu, Y. Katayama, and T. Miura, Electrochem. Solid-State Lett., 14, D110 (2011).

9. N. Tachikawa, N. Serizawa, Y. Katayama, and T. Miura, Electrochim. Acta, 53, 6530 (2008).

10. N. Serizawa, N. Tachikawa, Y. Katayama, and T. Miura, Electrochemistry, 77, 630 (2009).

11. N. Serizawa, Y. Katayama, and T. Miura, J. Electrochem. Soc., 156, D503 (2009).

12. N. Serizawa, Y. Katayama, and T. Miura, Electrochim. Acta, 56, 346 (2010).

13. R. Fukui, Y. Katayama, and T. Miura, J. Electrochem. Soc., 158, D567 (2011).

14. K. Fujii, T. Nonaka, Y. Akimoto, Y. Umebayashi, and S. Ishiguro, Anal. Sci., 24, 1377 (2008).

15. T. Torimoto, K. Okazaki, T. Kiyama, K. Hirahara, N. Tanaka, and S. Kuwabata, Appl. Phys. Lett., 89, 243117 (2006). 